\title{
INOVAR: VENTILADOR PULMONAR COM SENSORIAMENTO DE FLUXO PARA COVID-19
}

DOI: 10.37702/2175-957X.COBENGE.2021.3579

WILLIAN RICARDO BISPO MURBAK NUNES - willianr@utfpr.edu.br Universidade Tecnológica Federal do Paraná

Rua Agostinho Feijo Sanches 494

86079-420 - Londrina - PR

Vinicius Dario Bacon - viniciusbacon@utfpr.edu.br

Universidade Tecnológica Federal do Paraná

Rua Rolinhas 604

86700-110 - Arapongas - PR

Carlos Matheus Rodrigues de Oliveira - carlosoliveira@utfpr.edu.br Universidade Tecnológica Federal do Paraná UTFPR

Rua Cambuci 200

86802-060 - Apucarana - PR

Eduardo Costa Estambasse - eduardo.estambasse@bumoengenharia.com.br Bumo Engenharia e Manutenção

Rua Vigilato José da Cunha 190

86075-020 - LONDRINA - PR

Fernando Alves Negrão - fernandoanegrao@icloud.com

Pitágoras UNOPAR

Rua José Konhevalik 50

86189-095 - Cambé - PR

Edino Fernandes da Costa - edinocosta.2018@alunos.utfpr.edu.br Universidade Tecnológica Federal do Paraná Avenida Aviação 1936

86812-500 - Apucarana - PR

Marcelo Augusto Araujo de França - augustfranca@hotmail.com Universidade Tecnológica Federal do Paraná

Rua Versailles 357

86183-443 - Cambé - PR 
Luan Silvério Parro - luanparro@alunos.utfpr.edu.br

Universidade Tecnológica Federal do Paraná

Rua Maringá 95

86802-200 - Apucarana - PR

Lucas Zischler - luczis@alunos.utfpr.edu.br

Universidade Tecnológica Federal do Paraná

R. Silva Jardim 472

86811-480 - Apucarana - PR

Bruno Gabriel da Silva - bruno.1998@alunos.utfpr.edu.br

Universidade Tecnológica Federal do Paraná

travessa Ipiranga 65

86811-380 - Apucarana - PR

Leonardo Aparecido do Cati - leo-ducatti@hotmail.com

Universidade Tecnológica Federal do Paraná

Onorio Ramos da Silva 1002

15830-000 - Pindorama - SP

Rebeca dos Santos Monteiro - urebec@gmail.com

Universidade Estadual de Londrina

Rua Sapopema 312

86031-290 - Londrina - PR

Resumo: A pandemia devido ao vírus SAR-CoV-2 tem sido um dos desafios mais significativos para a humanidade nos últimos anos. Com o intuito de mitigar uma situação catastrófica no sistema de saúde, especialmente devido a quantidade limitada de número de ventiladores mecânicos, diversos pesquisadores propuseram alternativas de protótipos para ventilação mecânica. Este trabalho apresenta o InoVar, confeccionado em duas versões de protótipos. O projeto envolveu uma equipe multidisciplinar, constituída de docentes e alunos do curso de Engenharia Elétrica da UTFPR, campus Apucarana, bem como membros externos da sociedade civil. Resultados obtidos com os protótipos do Inovar são apresentados e discutidos. A solução desenvolvida é uma alternativa visando o registro nas agências reguladoras.

Palavras-chave: Sars-CoV-2, intubação, sistema respiratório, ventilação mecânica, ventilação de pressão positiva. 


\section{INOVAR: VENTILADOR PULMONAR COM SENSORIAMENTO DE FLUXO PARA COVID-19}

\section{INTRODUÇÃO}

Diante da escassez do número de ventiladores mecânicos, vários projetos opensource foram apresentados como alternativa para minimizar os efeitos do COVID-19. Em geral, todos equipamentos eletromédicos devem passar por processos de certificação e registro. No decorrer da pandemia, a ANVISA (2020) publicou resolução que definiu critérios e os procedimentos extraordinários para petições de regularização de equipamentos de proteção individual, de equipamentos médicos do tipo ventilador pulmonar e de outros dispositivos médicos. Com isto, ficou dispensado a certificação do INMETRO e agilizou temporariamente o processo de liberação de novos produtos registrados.

Diferentes desenvolvimentos de ventiladores artificiais pulmonares foram apresentados na comunidade científica, incluindo equipamentos de resgate ou transporte de pacientes. Todavia, deve ser ressaltado, que tais equipamentos necessitam de certificação e testes de acordo com as normas vigentes no Brasil.

Diversas universidades trabalharam em projetos de ventiladores de baixo custo e fácil acesso. Destaque para Escola Politécnica da USP, que desenvolveu o projeto INSPIRE, que é um ventilador econômico para produção em até duas horas, com custo em média 15 vezes mais barato que os ventiladores convencionais (INSPIRE, 2020; YAMAMOTO, 2020). Vale ressaltar ainda outros projetos brasileiros, tais como: GEPEC-VENT (UFRGS, 2020), InjeVent (UFSC, 2020), VExCO (CAMPOS, 2020), UNAMA (UNAMA, 2020), UEA/Honda (UEA, 2020), Fasten-Vita (KATEIVAS, 2020; RPC, 2020), Vent-U e COLLAB (UTFPR, 2020), Bold (BENETTI, 2020).

Grande parte dos projetos utilizam uma unidade manual de respiração, ou respirador manual, mais conhecido como "ambu". Segundo a ANVISA os equipamentos de suporte respiratório emergencial e transitório tipo "ambu automatizado", regulamentado pela Resolução RDC n. ${ }^{\circ} 386$, de 15 de maio de 2020, não podem ser comparados à complexidade da concepção e fabricação de um ventilador pulmonar. Esse equipamento possui finalidade de uso estritamente emergencial e transitória, com uso reservado para as situações em que não houver a disponibilidade de ventilador pulmonar para cuidados críticos ou ventilador pulmonar para transporte/emergência.

A Universidade de Caxias do Sul (UCS) juntamente com empresários desenvolveu o ventilador Frank 5010 (FRANK, 2020), cujo desempenho alcançou resultados satisfatórios e foi recentemente regularizado para comercialização. De maneira análoga, a empresa TACOM obteve com o VI-C19 do projeto INSPIRAR (FIEMG, 2020).

O grande desafio na área de ventilação pulmonar consiste em desenvolver equipamentos que garantam os requisitos mínimos de segurança para os pacientes. Isto significa que o projeto de um ventilador pulmonar deve ter um sistema de monitoramento, supervisão e controle das variáveis básicas de ventilação, tais como volume corrente ou volume inspirado, volume exalado, a pressão inspiratória de pico (Pip) e a pressão positiva expiratória final (PEEP, do inglês Positive End-Expiratory Pressure).

PEEP consiste na pressão alveolar acima da pressão atmosférica medida no fim da expiração. Corrobora neste sentido, a nota técnica Associação de Medicina Intensiva Brasileira - AMIB, no qual ressalta a necessidade de um ventilador mecânico possuir PEEP de 0 até $45 \mathrm{cmH} 2 \mathrm{O}$ para ser utilizado no tratamento de pacientes com COVID-19 objetivando redução dos danos pulmonares (BARBAS et al., 2013; AMIB, 2020). 
O principal efeito da PEEP é o de aumentar a capacidade residual funcional (CRF). Estudos "in vivo" feitos por Daly e colaboradores mostram que há um aumento linear no diâmetro alveolar com PEEP de 0 a $10 \mathrm{cmH} 20$, de 10 a $15 \mathrm{cmH} 20$ há um aumento menor do diâmetro e acima de $15 \mathrm{cmH} 20$ a pressão alveolar aumenta sem diferença mensurável no diâmetro alveolar.

Outrossim, destaca-se a necessidade do ventilador possuir um sistema eletrônico microcontrolado, realizando leitura de sensores e botões, monitorando carga da bateria, detectando falha da rede elétrica e de pressão do ar, bem como o acionamento de atuadores dos ramos inspiratório e exalatório, além de gerenciar informações para o usuário.

A interface homem-máquina (IHM) para um ventilador pulmonar, onde a equipe médica visualiza dados e registros do quadro clínico de um paciente e toma decisões importantes para evoluir o indivíduo. Outro elemento relevante é a estrutura do equipamento, que deve prover um suporte robusto para todo o sistema inserido e gerar mobilidade para sua transferência no ambiente hospitalar.

O projeto de extensão "InovAr - Ventilador Pulmonar" da UTFPR surgiu no início da pandemia para suprir itens de consumo na produção de ventiladores mecânicos, a citar por exemplo, sensor de fluxo. Este é um dispositivo indispensável para monitoramento do fluxo e o volume de ar inspirado pelo paciente. No entanto, no decorrer da pandemia seu suprimento se tornou escasso e de alto custo com a cotação do dólar. Neste sentido, professores da UTFPR campus Apucarana e parceiros externos formaram uma equipe multidisciplinar nas áreas de Engenharia Elétrica, Engenharia Mecânica e de Medicina, com destaque para um dos integrantes possuir expertise profissional em empresa de ventiladores mecânicos.

No final de abril de 2020, após resultados satisfatórios com o sensor de fluxo desenvolvido, a equipe decidiu montar um protótipo de ventilação mecânica, utilizando materiais acessíveis e com funcionamento já comprovado em aplicações industriais na área de automação e eletropneumática.

Figura 1 - InovAr VP1 (Cllique na imagem para visualizar a matéria).

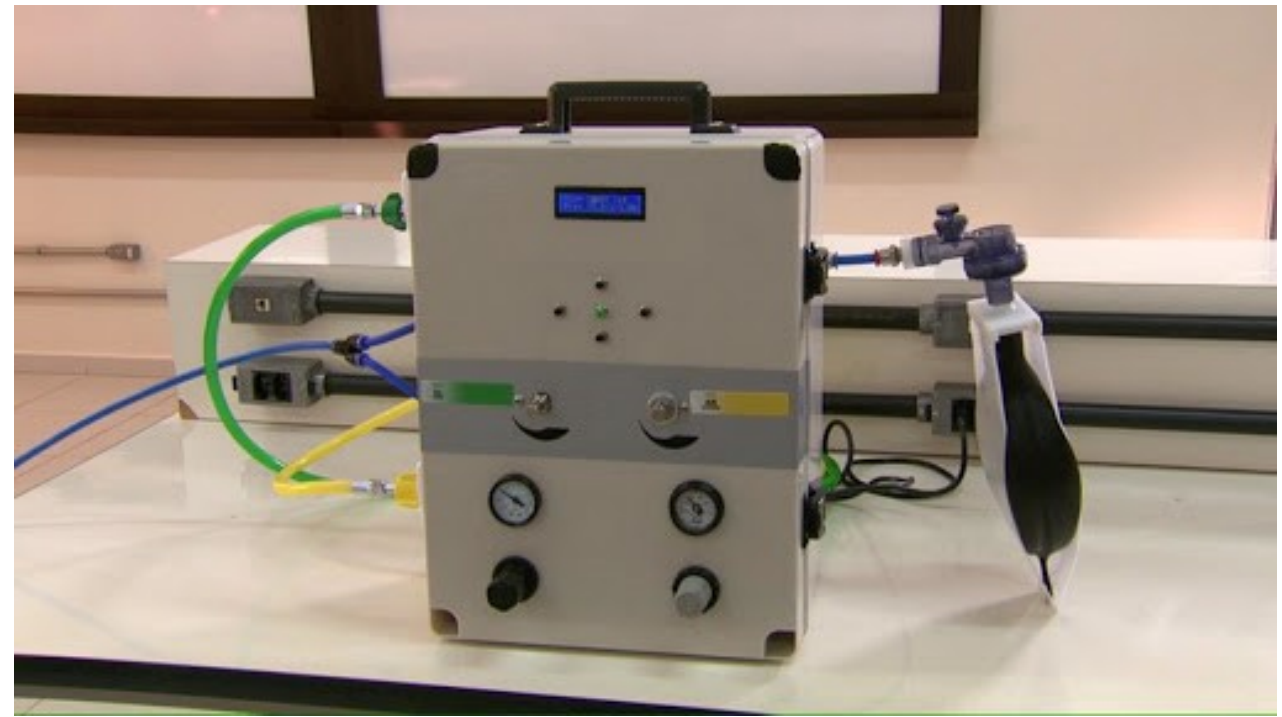

Fonte: Globo (2020). 
Em maio de 2020, a equipe apresentava um protótipo portátil, denominado InovAr VP1, a ser instalado com facilidade em ambientes hospitalares, com acesso a rede de oxigênio e ar, vide Figura 1. O projeto do equipamento previa o atendimento de pacientes em quadro grave devido ao COVID-19 ou outras doenças respiratórias. No desenvolvimento desta primeira versão a equipe já buscava atender os critérios previstos pela AMIB e os requisitos regulatórios da Associação Brasileira de Normas Técnicas (ABNT).

Em dezembro de 2020, a equipe apresentou o InovAr VP2, contendo alguns aprimoramentos comparado com a versão anterior. O InovAr VP2 se diferenciou por possuir um monitor de interface gráfica para supervisão, configuração dos parâmetros ventilatórios e uma estrutura metálica contendo rodízio de rodas para facilitar o deslocamento do equipamento no ambiente hospitalar.

Desde o início do projeto, a expectativa da equipe foi obter parceria estratégica com empresas da região norte do estado do Paraná, visando registrar o equipamento na ANVISA. A premissa para parceiros com o projeto InovAr foi o comprometimento de uma parcela de doações de ventiladores aos hospitais carentes da região. Ademais, o projeto previa também a possibilidade de utilização do equipamento por profissionais da área de saúde como ferramenta de treinamento em ventilação mecânica.

Neste artigo os resultados obtidos na montagem do ventilador pulmonar InovAr, em suas versões VP1 e VP2 são apresentados. Além disto, destaca-se a solução pneumática proposta para o ventilador, com itens acessíveis no mercado nacional para fins didáticos. A montagem e a validação do sensoriamento de fluxo são analisadas em diferentes cenários de teste. A estrutura contendo interface homem máquina (IHM) para o ventilador pulmonar são detalhados, visando a sua utilização em ambientes hospitalares. Por fim, destaca-se as ações realizadas e outros encaminhamentos obtidos para o desenvolvimento do projeto, visando a arrecadação de recursos para ensaios, parcerias de transferência tecnológica, mediante a premissa do compromisso na montagem, registro na ANVISA e doação de ventiladores pulmonares aos leitos de UTIs.

\section{MÉTODOS E PROCEDIMENTOS}

O projeto do ventilador pulmonar com sensoriamento de fluxo InovAr consistiu de uma solução eletropneumática, prevendo ramos de mistura de ar com oxigênio, controle das variáveis básicas de ventilação mecânica, tais como volume e percentual de mistura de ar inspirado, regulação da pressão inspiratória e expiratório do indivíduo.

Diante da complexidade de desenvolvimento de um ventilador pulmonar, o projeto InovAr foi dividido em áreas de atuação para acelerar sua prototipação. As áreas do projeto foram divididas em: sistema eletropneumático, sistema eletrônico, sistema estrutural e de interface gráfica.

O sistema eletropneumático consistiu no desenvolvimento do sensoriamento do fluxo de ar por pressão diferencial, especificação dos elementos pneumáticos de regulação, condicionamento, retenção e filtragem do ar comprimido.

O sistema eletrônico abrangeu toda a interface com o microcontrolador, por meio de circuitos de condicionamento dos sensores, acionamento dos atuadores pneumáticos, alarmes, interface com o usuário, supervisão e controle das variáveis de ventilação mecânica.

O sistema estrutural e de interface gráfica corresponde ao desenvolvimento de layout do ventilador, visando assegurar robustez, mobilidade no deslocamento do equipamento em ambiente hospitalar, bem como a elaboração de telas gráficas para a supervisão dos parâmetros ventilatórios. 


\subsection{Sistema eletropneumático}

O sistema pneumático do ventilador InovAr VP1 e VP2 correspondem aos elementos indicados na Figura 2. Em linha tracejada de cor vermelha está destacado o circuito de PEEP. Note que nesta proposta o sistema opera em malha aberta, ou seja, o ajuste de pressão desejada é realizado manualmente pela equipe médica no painel do ventilador e não há um controle automático para eventuais distúrbios de pressão. O sistema PEEP foi montado e sua validação ocorreu por meio de ensaios, mensurados por coluna d'água.

O sistema de sensoriamento de fluxo possui um principio de funcionamento baseado em diferencial de pressão. Ensaios com um medidor de fluxo foram realizados para caracterizar a relação entre as variáveis mensuráveis do dispositivo desenvolvido.

Também foi desenvolvido uma válvula pneumática servomecanizada para controle automático das variáveis de pressão e fluxo do ventilador.

Figura 2 - Diagrama completo indicando os

elementos do ventilador pulmonar InovAr.

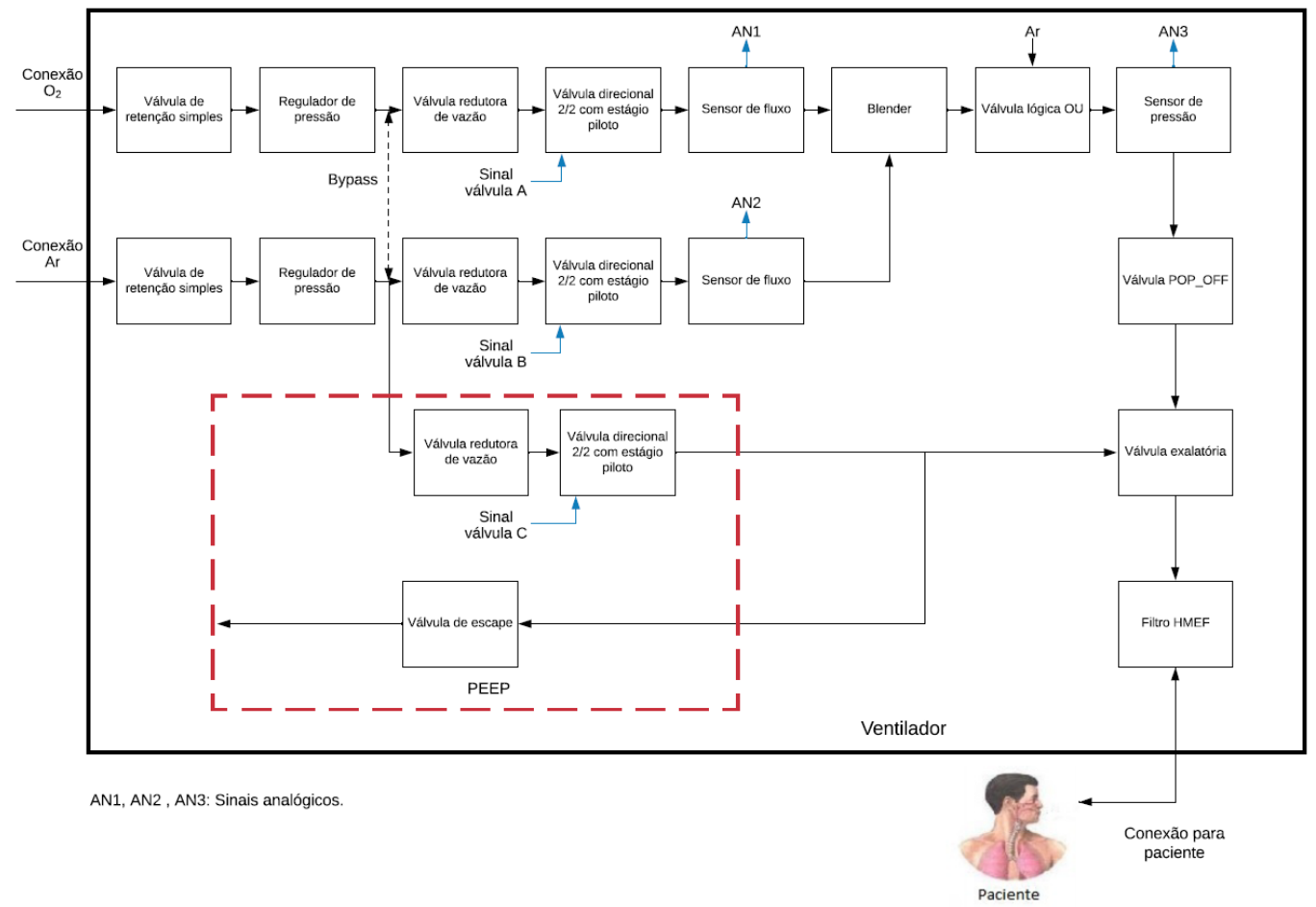

Fonte: Autores.

\subsection{Sistema eletrônico}

O sistema eletrônico é responsável pelo controle e supervisão de todas as variáveis do ventilador pulmonar. Para isto foi desenvolvido uma placa eletrônica, indicada na Figura 3, contendo circuitos de condicionamento de sinais para os sensores, drives para acionamento dos atuadores (válvulas solenóides, sinal sonoro e luminoso, entre outros elementos), leitura de botões do painel frontal, comunicação com a interface gráfica e um sistema microcontrolado para controle e automação dos processos. A placa desenvolvida também conta com um circuito capaz de prover energia ininterrupta por meio de bateria visando simplicidade e baixo custo, conforme indicado na Figura 3. Vale ressaltar que a interface gráfica do InovAr VP1 é por meio de um display de cristal líquido, enquanto do InovAr VP2 é um monitor. 
Figura 3 - Diagrama eletrônico do ventilador pulmonar InovAr.

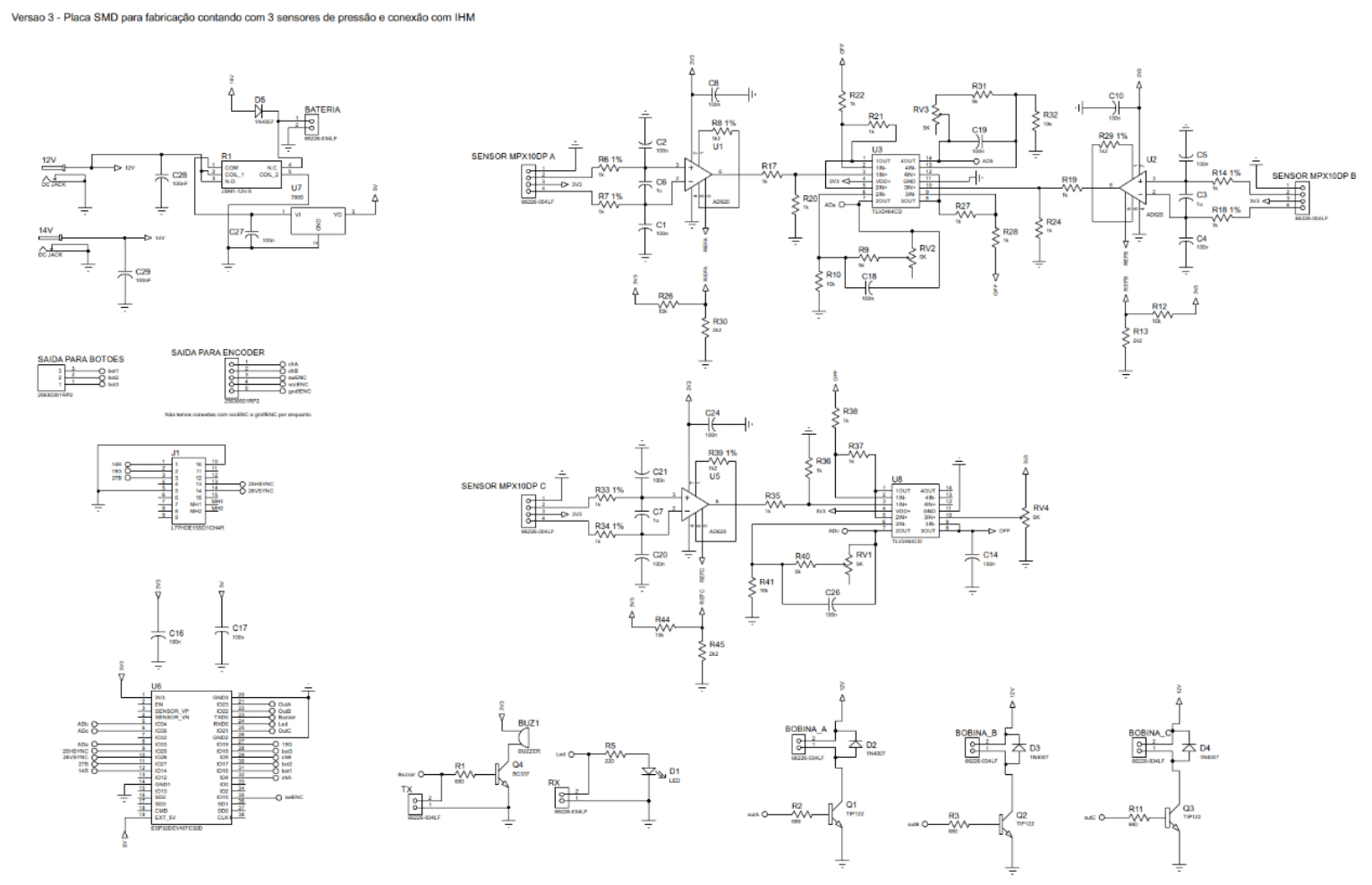

Fonte: Autores.

Ademais, foi proposto uma metodologia de ensaios para verificar a taxa de descarga e carga da bateria utilizada no protótipo e ainda avaliar o seu funcionamento avaliar o seu funcionamento diante de falha no fornecimento de energia pela rede elétrica.

Para o protótipo InovAr VP2 foi desenvolvida uma placa de circuito impresso contendo componentes SMD. Após conferência de cada um dos componentes presentes nos circuitos eletrônicos e seus respectivos encapsulamentos, os arquivos do layout da placa foram enviados para uma empresa especializada em impressões de PCB (do inglês, Printed Circuit Board). Após o término da confecção das placas, toda a montagem foi feita no laboratório da UTFPR.

\subsection{Interface homem máquina e estrutura física para mobilidade em ambiente hospitalar}

A interface homem máquina e a estrutura física em perfil de alumínio com rodas foram desenvolvidos para o InovAr VP2.

O código foi desenvolvido para ser embarcado no microcontrolador ESP32, com boa capacidade de processamento e com múltiplos núcleos, tendo em mente a necessidade renderização de uma interface gráfica em um monitor.

O sistema é dividido em algumas telas principais, cada uma definida por seu próprio thread, para otimização do processamento. O código completo desenvolvido para a interface gráfica está disponível no GitHub (github.com/luczis/Ventilador). 


\section{$3 \quad$ RESULTADOS E DISCUSSÕES}

\subsection{Sistema de sensoriamento de fluxo por diferencial de pressão}

O protótipo InovAr VP1 possui um painel de interface conforme indicado na Figura 4. Em sua vista frontal há uma IHM por meio de uma tela LCD e botões. Além disto, há acessíveis no painel reguladores de fluxo e pressão para ar comprimido e oxigênio.

O sistema permite o ajuste independente do fluxo provindo das linhas de ar comprimido e oxigênio. Em cada uma das linhas há o monitoramento do volume de ar por meio da pressão diferencial por um tubo sensor. Válvulas direcionais são acionadas em tempos específicos para a inspiração e expiração do paciente, conforme definido pelo usuário na IHM.

O ajuste necessário para a pressão PEEP é realizado pelo regulador de fluxo e da válvula de escape. A topologia adotada é do tipo malha aberta no qual o usuário realiza o ajuste de pressão necessária, mas não há um sistema de realimentação desta informação de forma a eximir possíveis erros de estado estacionário. Neste sentido, há possibilidade de operação em malha fechada com a utilização de válvulas proporcionais.

Figura 4 - Vista frontal do protótipo do ventilador pulmonar InovAr VP1.

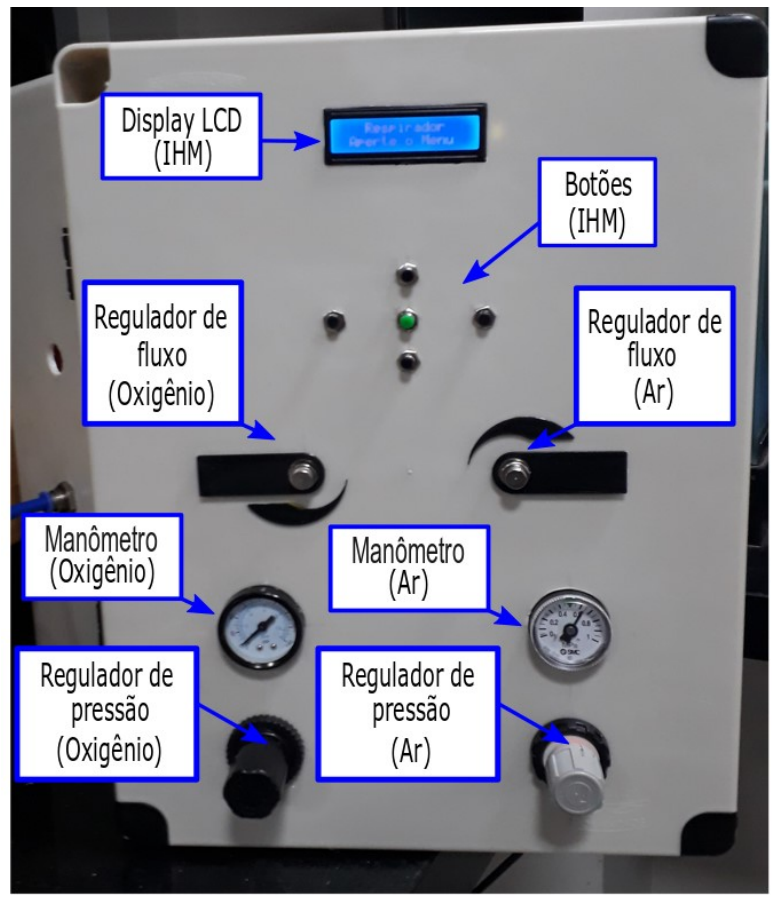

Fonte: Autores.

\section{Sensores de fluxo}

O sinal de pressão diferencial $\Delta \mathrm{P}$ provindo do sensor MPX10DP, após uma etapa de condicionamento eletrônico é digitalizado pelo microcontrolador cuja escala corresponde a valores no range de 0 a 1023. O fluxo de ar $Q$ dado em L/min foi mensurado por um fluxostato. Foram realizados ensaios com os dois tubos sensores.

A Figura 5 mostra um ensaio do tubo sensor com ajuste de fluxo de ar, sob pressão regulada em 4 bar na entrada do tubo. Note que o diferencial de pressão obtido no tubo sensor possibilita mensura o fluxo de ar em até $85 \mathrm{~L} / \mathrm{min}$. 
Figura 5 - Curva do diferencial de pressão $\Delta \mathrm{P}$ em função do fluxo de ar $\mathrm{Q}$ no tubo sensor, sob pressão regulada de 4 bar.

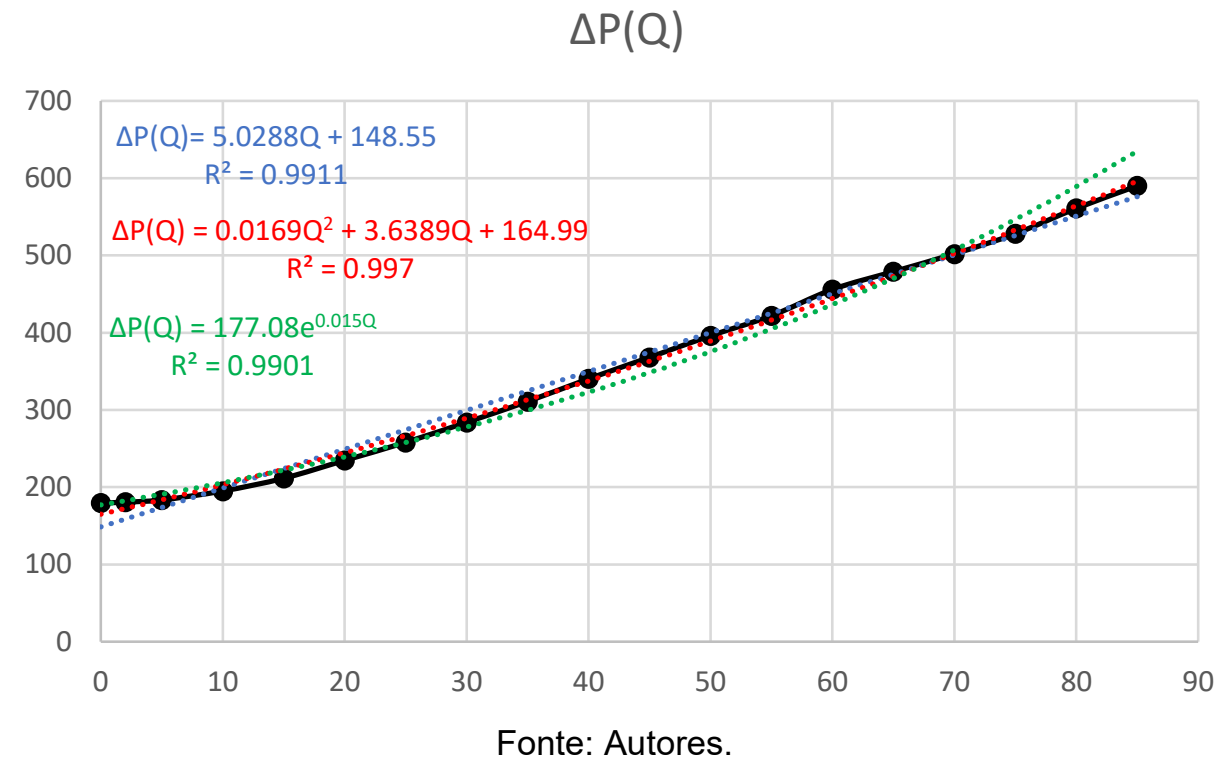

\subsection{Válvula de controle}

A Figura 6 ilustra a válvula de controle desenvolvida para restringir o fluxo de ar do sistema pneumático. Além disto, foi desenvolvido um encaixe para acoplamento do servomotor com a válvula em um compartimento de alocação.

O ensaio do sistema consistiu na avaliação do comportamento da servoválvula desenvolvida operando em malha aberta. Nesta operação o ar provindo de um compressor de ar foi inserido e regulado em pressão positiva de aproximadamente 1.0 bar, por meio de um regulador de pressão. Sob pressão regulada, o fluxo de ar foi ajustado nos ensaios por meio de um regulador de fluxo para uma região adequada de operação do sistema. Com o fluxo de ar ajustado realizou-se a restrição de ar por meio da servoválvula.

\subsection{Sistema de gerenciamento de energia}

Utilizando o esquemático apresentado na Figura 3 e do datasheet de cada um dos componentes, determinou-se a potência total do circuito. Com isto, obteve-se a carga mínima da bateria para suprir o circuito pelo intervalo de tempo de duas horas. Ou seja, assumindo os valores de potência total $P t$ igual a $128,844 \mathrm{~W}$, intervalo de tempo $\Delta T$ igual a 2 horas, conforme determinado pela AMIB (2020), e tensão nominal $V n$ igual a $12 \mathrm{~V}$, determina-se a capacidade total da bateria como sendo igual a 21,474 Ah.

Conforme o circuito da Figura 3, a bateria é conectada ao restante do circuito por meio de um circuito baseado em relê, diodos e capacitores. Durante a falta de energia o relê comuta para alimentar o circuito por meio da bateria, enquanto os diodos e capacitores são utilizados para manter o fornecimento de energia durante o tempo de comutação do relê. Para avaliar o circuito mediante interrupção da rede concessionária de energia, realizou-se testes experimentais que permitiram validar o fornecimento de energia ininterrupta, mesmo durante as transições de queda e retorno de energia por parte da rede concessionária. 


\section{COBENGE 2021 da ABENGE 28 a 30 de SETEMBRO

Figura 6 - Projeto e especificação de dimensões de caixa de suporte para acoplamento do servomotor com válvula esfera.

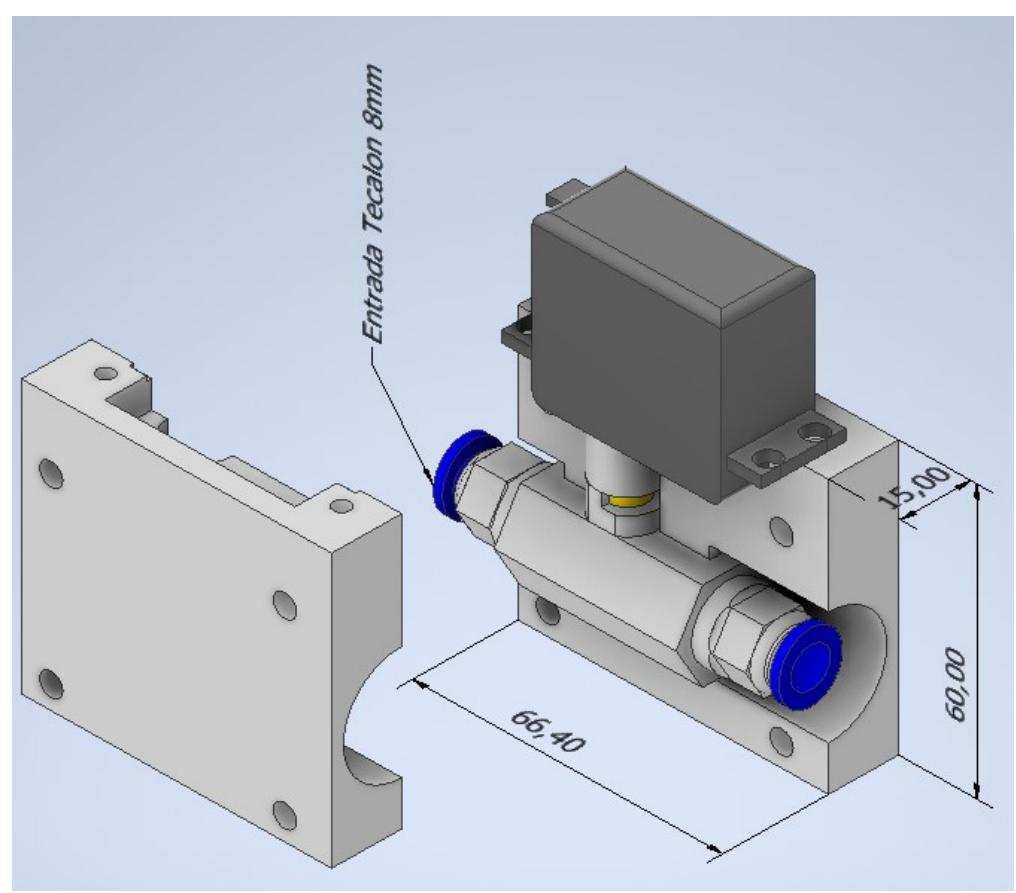

Fonte: Autores.

\subsection{Placa de circuito impresso}

O layout desenvolvido para a placa de circuito impresso está indicado na Figura 7.

Figura 7 - Placa de circuito impresso do ventilador InovAr Vp2: a) face superior; b) face inferior.
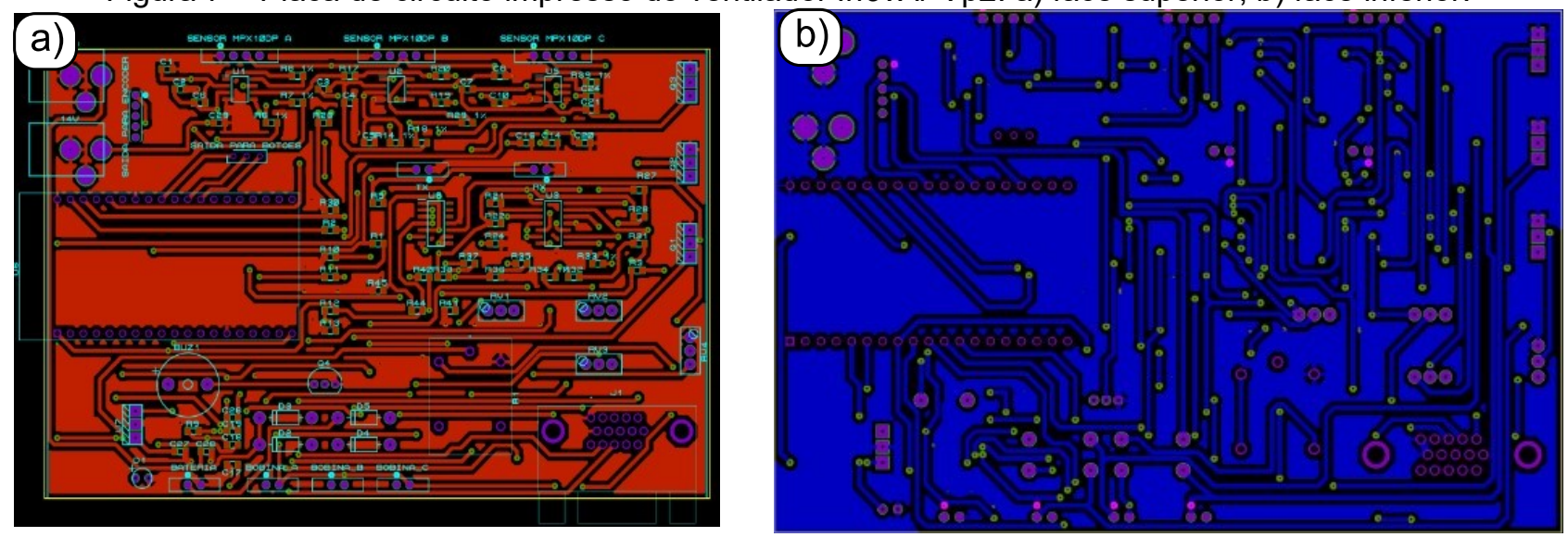

Fonte: Autores.

\subsection{Interface Homem Máquina (IHM)}

Com a placa de circuito impresso desenvolvida foi possível realizar o desenvolvimento da interface gráfica do ventilador InovAr VP2 por meio de um monitor. O InovAr VP1 possuía uma tela LCD alfanumérico, que impossibilita o acompanhamento e diagnóstico mais acurado do paciente por parte da equipe médica.

O propósito primordial da IHM para o InovAr VP2 é que fosse intuitiva ao usuário. Na tela inicial, vide Figura 8, uma chave seletora permite a mudança entre os parâmetros de configuração do tipo de paciente (pediátrico ou adulto), definição do peso, altura e modo de operação. Um encoder é utilizado para movimentar o cursor e alterar valores. $O$ valor com borda preta é a configuração que está definida, e que será passada para a próxima etapa. 
Figura 8 d) mostra a tela de solicitação para desligar o equipamento ou retorno para a tela principal. Para religar o equipamento pode-se utilizar o botão do encoder.

A interface gráfica foi embarcada em um ESP32, que possui um processador de dois núcleos com clock máximo de $240 \mathrm{MHz}$. A renderização de imagens por meio de processamento serial demanda um grande intervalo de tempo. Portanto, a renderização foi realizada apenas em pontos necessários, sempre utilizando sistemas de flags, impedindo que sessões do código sejam desnecessariamente reproduzidas.

A Figura 9 mostra o protótipo do ventilador pulmonar InovAr VP2 com a sua interface gráfica e sistema eletropneumático desenvolvido.

\section{CONCLUSÕES}

Durante a execução do projeto de extensão, a equipe do InovAr realizou soluções e inovações de elementos constituintes de um ventilador pulmonar.

O protótipo InovAr VP2 contemplou um sistema de controle e supervisão de variáveis da ventilação mecânica. A interface homem máquina desenvolvida mostrou-se estável, possibilitando a implementação em larga escala, reduzindo os custos de produção. A estrutura proposta permite mobilidade, garantindo robustez e segurança ao equipamento.

Algumas barreiras impediram o ventilador desenvolvido de alcançar o propósito fabricação e doação para hospitais carentes. O limitante principal correspondeu a falta de recursos para aquisição de materiais de consumo, instrumentos de medição e itens para ensaios de alto valor agregado visando registro na ANVISA. A equipe de pesquisa apresentou propostas para editais de fomento. Infelizmente, o InovAr não foi contemplado, principalmente pela exigência de propostas tendo empresas com certificações específicas para produção do equipamento.

O projeto foi divulgado junto à comunidade visando parcerias estratégicas com empresas. Entretanto, acredita-se que justamente o efeito da pandemia, possivelmente acarretou uma retração econômica e inibiu investimentos em projetos de inovação por parte de empresas da região.

Diante do exposto, fica evidente a necessidade de maior adesão e participação da comunidade universitária viabilizando o aporte financeiro para projetos de extensão. Também é necessário que existam equipes estratégicas nas universidades, atuando com empenho e expertise na divulgação e captura de empresas, parceiros da sociedade civil interessados nos projetos universitários.

Ao término de projeto, ficou explícito os fatos advindos da não adesão da sociedade civil em projetos como este. Poucos se sensibilizaram com a causa. Muitos se preocuparam consigo próprio e com a vida econômica de sua empresa. Esqueceram que para vencer esta batalha é necessário cooperação de todas as esferas da sociedade. Seja pública ou privada, da área da saúde ou da economia, todos devem olhar para o próximo. O número de mortos por COVID-19 aumenta a cada dia. Pessoas estão padecendo em leitos de enfermaria nas cidades grandes e pequenas, clamando por profissionais da saúde e leitos de UTI.

\section{Agradecimentos}

Agradecemos a Universidade Tecnológica Federal do Paraná pelo suporte financeiro para a execução deste projeto. Em especial a DIRGE-AP, DIREC-AP, COELT-AP, FUNTEF e colaborações provindas da sociedade civil. 
Figura 8 - Telas do ventilador: a) tela inicial de configurações; b) tela principal de supervisão das variáveis do ventilador; c) tela de reinicialização ou desligamento; d) tela de confirmação.
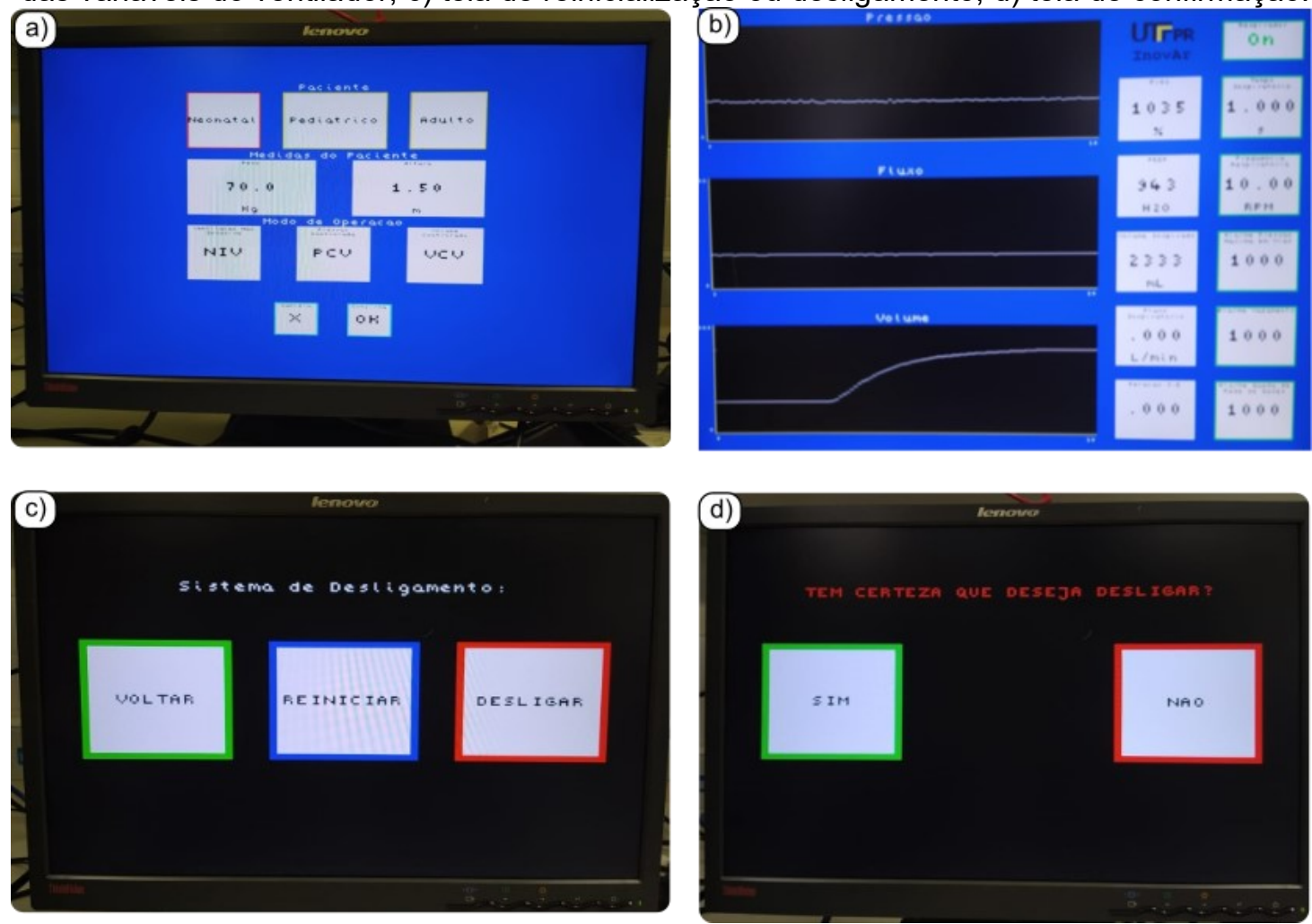

Fonte: Autores.

Figura 9 - Ventilador InovAr VP2.
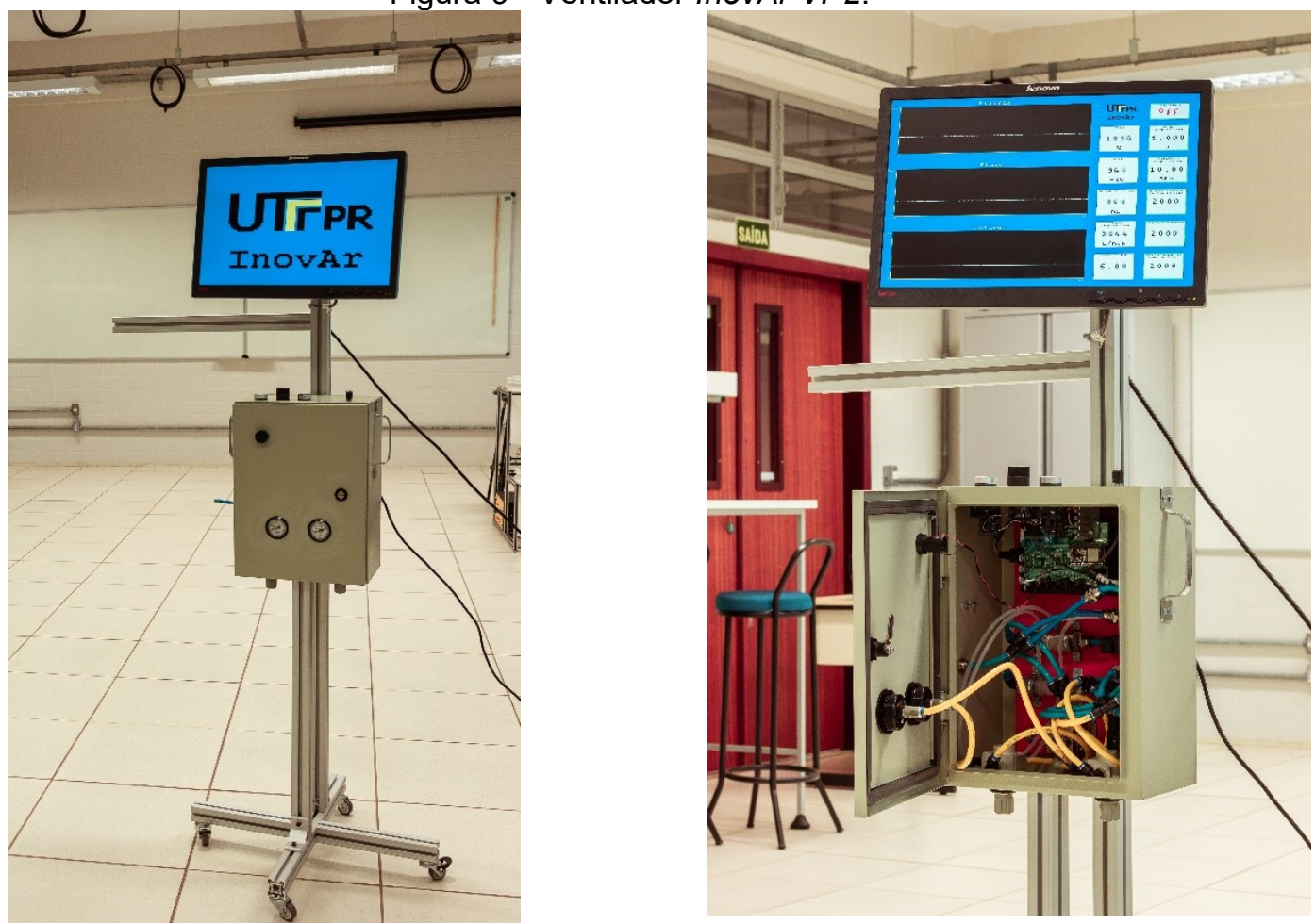

Fonte: Autores. 
REFERÊNCIAS

AMIB - ASSOCIAÇÃO DE MEDICINA INTENSIVA BRASILEIRA. Nota técnica sobre características de aparelhos ventiladores artificiais no suporte ao paciente com COVID-19. 2020. p. 2.

ANVISA - AGÊNCIA NACIONAL DE VIGILÂNCIA SANITÁRIA. Resolução de Diretoria Colegiada - RDC № 349, de 19 de março de 2020. 2020.

2001

. Resolução de Diretoria Colegiada - RDC N 185, de 22 de outubro de 2001.

BARBAS, C. S. V.; ÍSOLA, A. M.; FARIAS, A. M. C.; CAVALCANTI, A. B.; GAMA, A. M. C.; DUARTE, A. C. M. et al. Recomendações brasileiras de ventilação mecânica 2013. Parte I. Rev Bras Ter Intensiva. 2014;26(2):89-121.

Recomendações brasileiras de ventilação mecânica 2013. Parte 2. Rev Bras Ter Intensiva. 2014; 26(3):215-239.

BENETTI, E. Empresa de SC aguarda aval da Anvisa para fabricar ventilador pulmonar. NSC Total. Disponível em: https://www.nsctotal.com.br/colunistas/estelabenetti/empresa-de-sc-aguarda-aval-da-anvisa-para-fabricar-ventilador-pulmonar. Acesso em: 21 de dez. de 2020.

CAMPOS, A. C. Ventiladores pulmonares para covid-19 são testados com sucesso na UFRJ. Agência Brasil. Disponível em:

https://agenciabrasil.ebc.com.br/saude/noticia/2020-07/ventiladores-pulmonares-paracovid-19-sao-testados-com-sucesso-na-ufrj. Acesso em: 21 de dez. de 2020.

FRANK. Ventilador pulmonar FRANK 5010. Universidade de Caxias do Sul. Disponível em: https://www.frank5010.com.br/. Acesso em: 21 de dez. de 2020.

FIEMG. Respiradores produzidos pela Tacom, com apoio da FIEMG, são homologados pela Anvisa. Disponível em:

https://www7.fiemg.com.br/senai/Noticias/Detalhe/respiradores-produzidos-pela-tacomcom-apoio-da-fiemg-sao-homologados-pela-anvisa. Acesso em: 21 de dez. de 2020.

GLOBO. Coronavírus: Universidade desenvolve respirador de baixo custo no Paraná. RPC Curitiba. Disponível em:

https://g1 .globo.com/pr/parana/noticia/2020/05/23/coronavirus-universidade-desenvolverespirador-de-baixo-custo-no-parana.ghtml. Acesso em: 21 de dez. de 2020.

INSPIRE. Equipamento de suporte respiratório emergencial e transitório. Universidade de São Paulo. Disponível em: https://www.poli.usp.br/inspire. Acesso em: 21 de dez. de 2020.

KATEIVAS, M. Universidades desenvolvem ventiladores de baixo custo no Paraná, mas esbarram em burocracia e falta de recursos. Disponível em: https://g1.globo.com/pr/parana/noticia/2020/06/29/universidades-desenvolvemventiladores-de-baixo-custo-no-parana-mas-esbarram-em-burocracia-e-falta-derecursos.ghtml. Acesso em: 21 de dez. de 2020. 
UEA. Ventilador fabricado pela UEA e Honda apresenta resultado satisfatório no primeiro teste. Universidade do Estado do Amazonas. Disponível em: http://www2.uea.edu.br/home.php?dest=noticia\&notld=64285. Acesso em: 21 de dez. de 2020.

UFRGS. Ventilador pulmonar proposto pela UFRGS é de baixo custo, fácil montagem e simples manutenção. Disponível em:

https://www.ufrgs.br/coronavirus/base/ventilador-pulmonar-proposto-pela-ufrgs-e-debaixo-custo-facil-montagem-e-simples-manutencao/. Acesso em: $21 \mathrm{de}$ dez. de 2020.

UFSC. Professor da UFSC busca empresas para produção de ventilador pulmonar. Disponível em: https://noticias.ufsc.br/2020/05/professor-da-ufsc-busca-empresas-paraproducao-de-ventilador-pulmonar-aprovado-em-ensaios/. Acesso em: 21 de dez. de 2020.

UNAMA. UNAMA cria projeto de ventilador pulmonar de baixo custo. Universidade da Amazônia. Disponível em: http://www.unama.br/noticias/unama-cria-projeto-de-ventiladorpulmonar-de-baixo-custo. Acesso em: 21 de dez. de 2020.

UTFPR. Grupo de pesquisadores apresenta ventilador pulmonar de baixo custo. Universidade Tecnológica Federal do Paraná. Disponível em:

http://portal.utfpr.edu.br/noticias/geral/covid-19/grupo-de-pesquisadores-apresentaventilador-pulmonar-de-baixo-custo. Acesso em: 21 de dez. de 2020.

YAMAMOTO, E. Anvisa autoriza produção e doação do ventilador pulmonar Inspire. Disponível em: https://jornal.usp.br/institucional/anvisa-autoriza-producao-e-doacao-doventilador-pulmonar-inspire/. Acesso em: 21 de dez. de 2020.

\title{
INOVAR: LUNG VENTILATOR WITH FLOW SENSING FOR COVID-19
}

\begin{abstract}
The SAR-CoV-2 virus pandemic has been one of the most significant challenges for humanity in recent years. In order to mitigate a catastrophic situation in the health system especially due to the limited number of mechanical ventilators, several researchers have proposed prototype for mechanical ventilation. This work presents InovAr, made in two versions of prototypes. A multidisciplinary team formed by professors and students from the Electrical Engineering course at UTFPR, Apucarana campus, as well as external members of civil society carried out the InovAr. Results obtained with the prototypes are presented and discussed. The developed solution is an alternative for registration with regulatory agencies.
\end{abstract}

Keywords: Sars-CoV-2, intubation, respiratory system, mechanical ventilation, positivepressure ventilation. 